\title{
La participación infantil en los procesos migratorios. Las niñas y los niños de familias peruanas en Barcelona ${ }^{1}$
}

\author{
Iskra Pavez-Soto
}

Universitat Autònoma de Barcelona. Departamento de Sociología GEDIME (Grup d'Estudis d'Immigració i Minories Ètniques)

Iskra.pavez@uab.cat

Recibido: 16-06-2008

Aceptado: 14-12-2009

\section{Resumen}

En este artículo, se reflexiona sobre el cambio en las relaciones generacionales de las familias peruanas reagrupadas en Barcelona. A partir del concepto teórico de las niñas y los niños como actores sociales, se analiza la experiencia migratoria y la reagrupación familiar, con el objetivo de visibilizar su opinión acerca de los procesos migratorios familiares. Mediante entrevistas semiestructuradas, se explora la participación infantil en el proceso migratorio, los cambios en el ejercicio del poder generacional y la distribución del trabajo doméstico una vez que la familia se reúne en destino.

Palabras clave: relaciones generacionales, infancia inmigrante, reagrupación familiar, migración peruana.

Abstract. The participation of children in the migratory process. The children of Peruvian families in Barcelona

This article discusses the change in generational relationships of Peruvian families that have reunited within Barcelona. Based on the theoretical concept of children as social actor, the article analyses the migratory experience and reunifications of families in order to make their relevance for family migrations more visible. The participation of children in the migratory process, changes in the exercise of generational power and the distribution of domestic work once the family is reunited at its destination are explored through semistructured interviews.

Key words: intergenerational relationship, immigrant children, reunited families, Peruvian migration.

1. Este artículo es un avance de mi tesis doctoral de Sociología en curso, dirigida por la Dra. Sònia Parella Rubio en el Departamento de Sociología de la Universitat Autònoma de Barcelona. Quisiera agradecer a los evaluadores anónimos y a las personas que me han ayudado a mejorar la elaboración de este trabajo por sus valiosos aportes. 


\section{Sumario}

\section{Introducción}

2. Un nuevo enfoque para comprender a la infancia

3. La migración como una estrategia del grupo familiar
4. La participación infantil en el proyecto migratorio y en la reagrupación familiar

5. Las relaciones de género y generaciones en las familias peruanas de Barcelona

6. Conclusiones

7. Bibliografía

\section{Introducción}

Durante la década de 1990, llegaron muchas mujeres de Perú hacia España como pioneras del proyecto migratorio familiar. Algunas de ellas poseían altos niveles educativos, pero, dada la segmentación del mercado laboral, la mayoría se empleó en el servicio doméstico o en trabajos con poca calificación en Barcelona (Escrivá, 2000). Las familias peruanas que llegan a Cataluña proceden de diversos estratos sociales, la mayoría del sector medio y medio-alto y con diversidad de estructuras familiares (Altamirano, 2000). La feminización de las migraciones contemporáneas ha permitido comprender a los grupos familiares como verdaderas unidades de análisis, donde emergen de manera especial las niñas, los niños y los adolescentes como nuevos actores sociales participantes en dicho proceso migratorio familiar.

El objetivo de este trabajo es, por un lado, explorar la experiencia migratoria de la reagrupación familiar desde el punto de vista generacional, con énfasis en la perspectiva infantil, para conocer la vinculación transnacional de las familias durante el tiempo en que las niñas y los niños permanecieron en Perú al cuidado de otras personas mientras sus madres y sus padres estaban en Barcelona. En este sentido, intentamos analizar la motivación y la participación infantil en el proceso migratorio, específicamente en la reagrupación familiar. Por otro lado, nos planteamos el objetivo de reflexionar sobre la distribución generacional de los roles en el interior de las familias una vez reagrupadas en Barcelona. Intentamos profundizar en el ejercicio del poder y la autoridad adulta (expresada en permisos y sanciones) y la distribución y la responsabilidad del trabajo doméstico.

Para recoger la información, utilizamos la entrevista semiestructurada en profundidad. Esta técnica ofrece la dimensión biográfica y la percepción más íntima del proceso migratorio, al mismo tiempo que es un procedimiento que facilita la reflexión personal del sujeto entrevistado y nos aporta los significados que para él tiene la migración. La metodología cualitativa nos ayuda a conocer en profundidad un fenómeno, la intención es adentrarnos en la comprensión que los sujetos tienen sobre sus vivencias. No se pretende generalizar a partir de los casos estudiados. Se entrevistaron a dos niñas y a cinco niños desde los doce hasta los diecisiete años de edad, la mayoría de los cuales asistía a la educación secundaria obligatoria. También se entrevistaron a siete madres y a un padre, la mayoría con niveles de estudios medios y algunos supe- 
riores, quienes desempeñaban ocupaciones de limpieza, cuidado y seguridad. Las familias llevan de uno a seis años en Barcelona. Los criterios de selección de las personas participantes, además de ser peruanas y llevar residiendo como mínimo un año en Barcelona, era haber vivido procesos de reagrupación familiar. El número de catorce entrevistas responde a la etapa exploratoria en que se encuentra el trabajo de campo de la tesis doctoral, y por lo mismo todos los resultados tienen este carácter.

Los contactos se realizaron con organizaciones peruanas establecidas en Barcelona aplicando la técnica de bola de nieve, lo que consistió en localizar a algunos individuos con las características descritas, los cuales nos condujeron a otros y así sucesivamente, como un efecto de bola de nieve. La ventaja de dicha técnica es que nos aporta información de primera fuente, porque se contacta con las personas que interesan particularmente para este estudio, pero tiene la desventaja que puede haber un sesgo en la selección de los participantes y ello afectar a la confiabilidad.

Para alcanzar los objetivos, se considera a las niñas y a los niños como sujetos con una visión propia sobre lo que se estudia, con lo que se garantiza su derecho a participar e informarse. En primer lugar, las entrevistas se contactaron con las madres y los padres, a quienes se les entregó la información sobre la entrevista a realizar y se les pidió su consentimiento. En segundo lugar, se informó a las niñas y a los niños sobre el proceso y los objetivos de la investigación, los usos y la confidencialidad de los datos recopilados, así como la posibilidad de participar libremente en la entrevista, y también su derecho a negarse, para lo cual firmaron un formulario de consentimiento (Laws y Mann, 2004).

En los apartados siguientes, se revisan algunos conceptos respecto a la comprensión teórica de la infancia y su participación en las migraciones y en la reagrupación familiar.

\section{Un nuevo enfoque para comprender a la infancia}

Desde un punto de vista histórico, podemos decir que la infancia siempre está presente «invisiblemente» en los grandes sucesos del «mundo adulto». La idea moderna de las niñas y los niños unidos a la institución familiar y escolar es relativamente reciente, tanto en Europa como en Sudamérica. Anteriormente se daban otros modelos familiares, como la familia extendida, y su participación laboral constituía un aporte a la economía familiar. El surgimiento, a inicios del siglo XX, de la idea vigente sobre la infancia coincide con el desarrollo de programas asistenciales y religiosos de atención a las niñas y a los niños excluidos, unido a los procesos de escolarización obligatoria masiva (Ariés, 1987). Esta idea de la niñez como una construcción cultural e histórica es una de las premisas de la sociología de la infancia, la cual intenta estudiar específicamente el quehacer infantil de las niñas y los niños, en una zona intermedia entre la sociología de la educación y de la familia (Gaitán, 2006b, 2008; Rodríguez, 2007).

En cada espacio y en cada tiempo presente, las niñas y los niños son actores sociales, aunque actúen «invisibilizadamente» en muchas actividades sociales y 
familiares, por ejemplo: en los procesos migratorios iniciados y legislados por las personas adultas. Como grupo social, están en permanente relación y negociación con los demás grupos sociales. Sin embargo, esta actuación pocas veces es reconocida por la colectividad, principalmente por su situación de dependencia económica y física durante los primeros años de vida, lo que les lleva a una subordinación y paternalización permanente (Gómez-Granell y García-Milà, 2004; Liebel, 2007). Según Lourdes Gaitán (2008), para que las niñas y los niños que participan en procesos migratorios familiares sean sujetos sociales, es preciso que se den dos condiciones: por un lado, que desarrollen acciones e incidencia en su propia vida y en el entorno que habitan, y, por otro, que dicha acción social sea reconocida por los demás actores sociales, tanto por su familia, como por las instituciones de la sociedad de origen y de destino. Sin embargo, esta última condición es difícil que se cumpla, porque, en general, se les trata como un «objeto" pasivo y receptor de las decisiones y de la protección adultas, y no como un sujeto que sí actúa dentro del marco minoritario de poder que tienen para hacerlo.

No obstante, la infancia como categoría está presente de todos modos en nuestra estructura social, puesto que es una parte que existe permanentemente, aunque sus miembros se renueven de manera constante, así como la juventud. De este modo, también es una categoría sujeta a los cambios socioeconómicos y a las transformaciones culturales que afectan a los modos específicos de «ser niña o niño inmigrante» en cada contexto (Gaitán, 2006b, 2008).

Pero la infancia en general no es un grupo social homogéneo. Particularmente dentro del grupo infantil inmigrante también existen desigualdades de género, de clase, de nacionalidad y de origen cultural o religioso. Las características del proyecto migratorio familiar marcan las experiencias y las expectativas infantiles. El retorno, la permanencia definitiva o la reagrupación son elementos que se revisan continuamente en el interior de las familias y transforman la propia experiencia migratoria infantil (Pedone, 2007).

Para entender la actuación social infantil, es preciso identificar las relaciones y las negociaciones que se llevan a cabo con los otros actores del proceso migratorio familiar. Las niñas y los niños desarrollan relaciones de poder con los demás miembros de la familia, puesto que cada cual ocupa una determinada posición y un espacio generacional (Gaitán, 2006b).

En nuestra investigación, consideramos a la «infancia» como un espacio social, jurídico, económico y cultural que comparten los individuos que aún no han cumplido los dieciocho años (Gaitán 2006a, 2006b), aunque existen importantes diferencias de edad dentro del grupo infantil, ya que, a medida que las personas van creciendo, adquieren nuevos derechos y responsabilidades -lo que se denomina jurídicamente "autonomía progresiva»— respecto a trabajar legalmente, contraer matrimonio o la libertad para tomar las propias decisiones. Sin duda, las niñas y los niños experimentan determinadas desigualdades, exclusiones o privilegios según la edad o la etapa escolar en la que se encuentren, puesto que, por ejemplo, los bebés necesitan protección, pero tienen menos libertad de movimiento, mientras que las adolescentes y los adolescentes pueden ejercer mayor autonomía respecto a las decisiones y a la volun- 
tad adulta, y por lo mismo se flexibiliza su protección. De todas maneras, los dieciocho años representan una frontera generacional. Las niñas, los niños y los adolescentes comparten dicha pertenencia generacional frente a las personas adultas, a quienes se les considera como tal cuando cruzan esa frontera construida socialmente en la actualidad, pero basada en procesos biopsicosociales (aunque la juventud tiende a difuminar a veces dicho límite).

Las niñas y los niños migrantes forman parte de la infancia y de la migración a la vez, dos fenómenos sociales que les sitúan como una generación histórica específica respecto a sus madres, a sus padres y a otros parientes adultos que también participan en los procesos migratorios.

Según Mannheim (1928), la generación es una experiencia histórica que crea un marco común de vivencias e interpretaciones durante la juventud - en nuestro caso, durante la infancia-, puesto que facilita una comprensión del mundo similar. Ciertamente la migración es un punto de referencia que comparten sus protagonistas. Tanto el momento político, social y económico (las redes en que participa un grupo humano), como los patrones relacionales forman parte de "la experiencia migratoria» de cada cohorte generacional (Eckstein, 2002). De este modo, la edad se constituye en una categoría básica de diferenciación y análisis sociológico de los hechos sociales en general y de los procesos migratorios familiares en particular (García Borrego, 2008).

\section{La migración como una estrategia del grupo familiar}

A nivel analítico, podemos decir que la familia constituye un verdadero observatorio de las relaciones de poder generacional y de género que viven sus miembros, de acuerdo con las pautas vigentes en cada sociedad. Es el lugar donde la división sexual del trabajo se palpa, puesto que las mujeres — madres o no- son las responsables de realizar las labores asociadas a la reproducción social, específicamente la limpieza y el cuidado. Las niñas y los niños llevan a cabo su aporte haciendo su trabajo escolar y doméstico como contribuyentes no reconocidos de «bienestar familiar» (Gaitán, 2006b), sobre todo las niñas y las adolescentes, a quienes se les asignan determinadas tareas por su pertenencia de género.

Una vez que se comienzan a estudiar las desigualdades de género en la migración, se desvelan otros aspectos familiares y sociales que habían quedado ocultos, como el cuidado infantil, la inserción escolar y social de las niñas y los niños, las relaciones de pareja, el impacto de las remesas y los vínculos transnacionales de asistencia, entre otros temas (Pedone, 2003; Carrasco, 2004a, 2004b; Parella, 2007; Solé et al., 2007). Los estudios sobre las niñas y los niños de origen extranjero, tanto en Estados Unidos como en Europa ${ }^{2}$, se han cen-

2. En el ambiente académico de estos contextos, existe un creciente debate conceptual sobre cómo denominar a la infancia inmigrante o la llamada «segunda generación». Aparicio y Tornos (2006) apuestan por un uso conceptual más histórico que biológico del término «segunda generación», dado que la migración está inscrita en un marco histórico específico, a la vez que se vive en un ámbito intrafamiliar particular. 
trado principalmente en los resultados escolares y laborales, el uso lingüístico y las estrategias de movilidad (Portes, 1996; Portes y Rumbaut, 2001; Portes et al., 2006; Aparicio y Tornos, 2006), considerando la discriminación racial, la segregación habitacional, las políticas migratorias y los cambios en la economía (Levitt y Waters, 2002; Suárez-Orozco y Suárez-Orozco, 2003; Carrasco, 2004a; García Borrego, 2008).

En los estudios sobre migración y niñez (Suárez Orozco y Suárez-Orozco, 2003; Pedone, 2007; Gaitán, 2008), la participación infantil en los procesos migratorios familiares se observa con cierta ambivalencia, porque en algunos casos no se consulta la opinión a los niños ni a las niñas y se les trata como objetos, pero en otros pueden ser altamente considerados, con gran responsabilidad y capacidad de decidir e incidir en el proyecto migratorio familiar. En general, los proyectos migratorios tienen como fin último el bienestar infantil, pero a veces las niñas y los niños ni siquiera son consultados sobre estas cuestiones y viven los cambios y las pérdidas de la migración, de manera muy solitaria.

En el caso específico de la migración peruana en España, las motivaciones para la reagrupación de las hijas y los hijos en destino tienen una dimensión afectiva, además de las expectativas educacionales. En ellos se depositan muchas expectativas personales que las madres y los padres no han podido conseguir en destino (Labrador, 2001: 149; Aparicio y Tornos, 2006; Solé et al., 2007).

Cuando se lleva a cabo la migración adulta y las hijas y los hijos quedan en origen, tradicionalmente las abuelas o las tías asumen la responsabilidad del cuidado infantil, aunque las madres y los padres participan desde la distancia en la gestión doméstica, mediante la asistencia económica a través de las remesas, la toma de decisiones conjuntas y el intercambio de información permanente. Es lo que se denominan «vínculos transnacionales de cuidado» (Bryceson y Vuorela, 2002; Solé et al., 2007). Las relaciones generacionales cambian durante este periodo, puesto que las formas de comunicación, el ejercicio de los roles de cuidado y la autoridad se comienzan a experimentar de nuevas maneras (Pedone, 2007; Parella, 2007; Solé et al., 2007). En algunas familias, a las hijas — y, en menor medida, a los hijos — mayores les toca asumir roles de cuidado con sus hermanas y sus hermanos más pequeños (Pedone, 2003; Gaitán, 2008). Algunos pueden vivir este periodo de vinculación transnacional como algo traumático o como un abandono, si no participan en la elaboración del viaje, desconocen las fechas de reencuentro, mantienen una relación poco saludable con quienes les cuidan en origen o tienen una escasa comunicación filial. Por el contrario, si el proceso es vivido con planificación y participación infantil, puede ser algo doloroso pero aceptable, puesto que las hijas y los hijos van a contar con el apoyo permanente de otras personas que les acompañan en origen (SuárezOrozco y Suárez-Orozco, 2003: 123; Pedone, 2003, 2007; Parella, 2007).

La reagrupación familiar lleva consigo diversas emociones para las niñas y los niños, porque dejan a sus seres queridos que les cuidaban en origen - normalmente, las abuelas o las tías-, pero también tienen las expectativas de viajar y reunirse con sus madres y sus padres que no han visto, a veces, desde hace años (Suárez-Orozco y Suárez-Orozco, 2003: 125). Por su parte, las personas 
adultas deben, necesariamente, re-aprender a dialogar, a negociar las vicisitudes diarias y a enfrentarse a ellas, porque las formas de ejercer su poder y la participación infantil se han transformado (Pedone, 2003, 2007; Terren y Carrasco, 2007). Es un proceso de re-conocimiento mutuo, porque ambas partes necesitan dialogar para re-legitimar y re-construir los lazos filiales sin caer en la indiferencia o en la frustración (Suárez-Orozco y Suárez-Orozco, 2003: 136; Solé et al., 2007).

\section{La participación infantil en el proyecto migratorio y en la reagrupación familiar}

En este apartado, se aplican los conceptos teóricos anteriormente presentados en el análisis de las entrevistas de las familias participantes en nuestra investigación, cuyos objetivos se van configurando en categorías de estudio. Nos proponemos reflexionar sobre la experiencia migratoria de las niñas y los niños mientras permanecen en Perú a cargo de una figura adulta de cuidado infantil, así como su vinculación transnacional con sus madres y sus padres que ya están en Barcelona; además, se analiza la motivación y la participación de las hijas y los hijos en estas cuestiones.

La figura de cuidado infantil en Perú asume una gran responsabilidad en esta tarea y también desarrolla un importante vínculo de apego. En nuestras entrevistas, se constata que, cuando las niñas y los niños tienen que reunirse en Barcelona con sus progenitores, el distanciamiento de la abuela o de la tía que les cuidaba desalienta el traslado, porque están afectivamente vinculados con estas personas. Generalmente, las madres y los padres que están en el extranjero apoyan la función del cuidado infantil a través de la comunicación permanente y el envío de las remesas monetarias para la subsistencia, de este modo, se lleva a cabo la vinculación transnacional en las familias peruanas participantes en este estudio. Según el discurso infantil, se solicita dinero o materiales escolares cuando se necesitan realmente y no para manipular o conseguir objetos de consumo. Más bien son las madres y los padres quienes utilizan el envío de regalos y las "propinas» como una forma de estar presentes simbólicamente en Perú. Son ellos quienes envían dinero y determinados objetos, pero también les traspasan valores y nuevas creencias de la vida en Barcelona, lo que Levitt y Waters (2002) denominan «remesas sociales».

Me parecía que íbamos a tener una vida, bueno en ese tiempo no lo pensaba tan así. Decía, antes de venir, que ¿para qué veníamos? Sabía que era para tener una vida mejor que la que teníamos [...] Yo no quería venir a España, pero no lo dije tampoco. Porque no quería separarme de mi familia, de mis amigos. Toda la vida la tenía allí, y aquí era comenzar de nuevo, como si volvieras a nacer. (Julieta, 13 años)

Según algunos estudios (Pedone, 2003; Carrasco, 2004a, 2004b; Terren y Carrasco, 2007), las familias normalmente prescinden de la opinión infantil 
a la hora de elaborar sus proyectos migratorios, ya que las personas adultas centran sus esfuerzos en organizar un sinfín de detalles y un proceso participativo podría ralentizarlo todo aún más. Pero en nuestras entrevistas vimos que, en algunas ocasiones, las niñas y los niños sí son consultados sobre el proyecto migratorio familiar. Cuando las personas adultas facilitan un proceso participativo dando la posibilidad de opinar y decidir, las hijas y los hijos se sienten más responsables y también protagonistas de este plan. En efecto, son parte de este plan y sus vidas se transforman con esta decisión, por lo tanto, es lógico que también opinen.

De acuerdo con lo que se expuso anteriormente, los estudios de SuárezOrozco y Suárez-Orozco (2003) han demostrado que la implicación de todos los actores en la decisión migratoria contribuye a fortalecer los vínculos filiales, que necesitan recomponerse una vez reagrupado el núcleo. En nuestras entrevistas, se constata que la reagrupación es una parte importante del proyecto migratorio. Las niñas y los niños tienen variadas motivaciones para viajar, desde la esperanza de volver a estar junto a sus progenitores, hasta las expectativas de estudiar y conocer un nuevo país muchas veces idealizado.

Sí, me preguntaron la opinión y recuerdo que dije que sí, que estaba de acuerdo y que, si era para mejor, pues que se haga, ¿̨no? (Eduardo, 17 años)

Las niñas y los niños peruanos en Barcelona participantes en esta investigación continúan vinculados con algunas personas en Perú, principalmente con sus familiares, como la abuela o la tía que les cuidaba, con quienes se comunican por teléfono; así como con sus primas, sus primos y sus amistades de la misma edad, con quienes se relacionan a través de Internet. En algunas ocasiones, viajan a Perú durante las vacaciones o para la celebración de determinadas fiestas (por ejemplo: la fiesta de los quince años es muy importante, porque marca una frontera generacional para las niñas, quienes en ese momento comienzan a ser consideradas como «mujeres»). El desplazamiento se realiza con una frecuencia muy relativa, ya que está sujeto al presupuesto económico.

Llamo todas las semanas, una vez a la semana o dos veces así, pero sí hablo con ella [la abuela]. Me comunico por Internet con mis primos más que todo, como ellos van a Internet y eso, yo hablo con ellos por ahí [...] No he viajado, pero el viaje es para junio más o menos, este verano. (Víctor, 13 años)

Como se puede apreciar, el dominio y el uso de la tecnología entre el grupo de iguales es una forma de comunicación característica de las nuevas generaciones de inmigrantes, lo que Levitt y Waters (2002) denominan las «características generacionales de cada cohorte migratoria», es decir, las influencias del contexto cultural en el cual se vive la migración familiar.

Por otro lado, un tipo de participación infantil muy particular en el proyecto migratorio se refiere al aprendizaje de las lenguas de los lugares de des- 
tino. Según Portes y Rumbaut (2001), esto permite que las niñas y los niños obtengan información y posibilidades de socialización muy diferentes a las de sus madres y sus padres, quienes aprenden el idioma con dificultades y a veces sólo en un nivel básico. La situación bilingüe específica de Barcelona admite introducir algunos matices en la formulación teórica de Portes y Rumbaut, respecto a las implicancias del dominio lingüístico en la familia. Las niñas y los niños peruanos entrevistados en nuestro estudio aprenden el catalán en el sistema educativo, lo que sin duda les facilita relacionarse con la infancia autóctona y acceder a diferentes fuentes de información. Ellos nos dicen que hacen uso del catalán en el ámbito familiar cuando surgen situaciones que precisan traducción, por ejemplo: cuando reciben correspondencia institucional (documentos bancarios, escolares, etc.) o cuando ven alguna publicidad y oyen frases en la calle que no entienden sus madres y sus padres u otros adultos de la familia. En dichos casos, ellos hacen el papel de traductores. Mayoritariamente, las niñas y los niños que saben el idioma sólo lo hablan en el colegio.

Sí, hablo catalán; sí, lo entiendo. Bueno, hablo en la clase de catalán, sólo en la clase de catalán, porque no me gusta el catalán, lo hablo, pero a veces. A mis padres les traduzco cosas del cole, en la calle cuando me preguntan «¿qué quiere decir?». Cuando ellos me lo piden, yo se los traduzco, cuando necesitan ayuda en algo [...] Me siento más tranquila porque lo entiendo y lo sé traducir, cuando necesito leer y me sirve. (Julieta, 13 años)

El conocimiento infantil del catalán otorga algunos beneficios a quienes lo saben, pero no alcanza a invertir los roles tradicionales de autoridad. Es lo que Portes y Rumbaut (2001) llaman «aculturación disonante», puesto que las madres y los padres hablan castellano, un idioma que también les permite trabajar y socializarse en la ciudad. A partir de la información cualitativa obtenida en nuestras entrevistas, se denotan relaciones de cooperación en cuanto al intercambio lingüístico, en el sentido que las hijas y los hijos ayudan a sus progenitores a traducir lo que no saben.

\section{Las relaciones de género y generacionales en las familias peruanas de Barcelona}

A continuación, se desarrolla el segundo objetivo de la investigación planteado en la introducción respecto a los cambios en las relaciones de género y generacionales que experimentan las familias peruanas entrevistadas en este estudio y que participan en procesos de reagrupación familiar. Para lograr este propósito, presentamos la información extraída de las entrevistas infantiles y adultas ordenadas en tres categorías de análisis: el ejercicio del derecho a la opinión infantil; la autoridad adulta manifestada en los permisos y en las sanciones, y, por último, las transformaciones en la distribución y la responsabilidad del trabajo doméstico. 
Una de las dimensiones del poder generacional se refiere a que las personas adultas "permitan hablar» y escuchen a las niñas y a los niños en los asuntos migratorios y familiares, cuestiones que ineludiblemente les afectan. A partir de nuestras entrevistas, se deduce que las madres y los padres abren paso a la opinión infantil en variados asuntos y decisiones familiares.

Entre yo y mi marido, los dos, lo conversamos cualquier cosa y, bueno, lo decidimos, y cuando es alguna cosa que tenga algo que ver con él [con el hijo] sí lo hablamos con él, cuando es alguna cosa que solamente nos concierne a los dos, que te digo en cuestión del piso y eso, solamente los dos. Pero cuando tenemos que pedirle su opinión en alguna otra cosa que le va a afectar a él, sí que se la pedimos. (Cecilia, 44 años)

Según Gaitán (2006b) y Gómez-Granell y García-Milà (2004), el ámbito familiar puede ser un espacio de solidaridad y negociación permanente entre los miembros del grupo. Las madres y los padres peruanos entrevistados en nuestro estudio tienen variados relatos respecto a permitir y a considerar la opinión infantil. Desde el punto de vista de las niñas y los niños que entrevistamos, ellos sí sienten que son consultados en la práctica sobre diversos temas, como las salidas familiares, los viajes a Perú o la cantidad de dinero que se envía a través de las remesas.

Doy mi opinión de todo, ella [la mamá] todo me lo pregunta. Por ejemplo, de cuánto tiene que mandar a Perú, si está bien el dinero [...] [Pero] la opinión a veces la toma en cuenta, y a veces no. (Beatriz, 15 años)

A partir de los relatos de nuestras entrevistas, vemos que las hijas y los hijos perciben que son tomados en cuenta, que pueden opinar e incidir en las decisiones. En otras palabras, que sus ideas finalmente son consideradas en el ámbito familiar.

A veces, conforme si es importante, muy importante, lo eligen entre ellos dos. Si es algo así, pues me preguntan, como para comprar algo, como la televisión, la radio, algo, pero cosas así normales. Pues no me preguntan si tienen que cambiar de banco, esas cosas raras. (Juan, 14 años)

Otra dimensión del poder generacional se refiere a la facultad de dar permisos o de imponer castigos sobre las niñas y los niños. Las personas adultas que realizan el acompañamiento infantil establecen ciertas libertades y sanciones frente a determinadas situaciones familiares que ellas consideran deben ser sancionadas o no. De acuerdo con nuestras entrevistas, la capacidad de dar permisos a las niñas y a los niños es una función que comparten los miembros adultos de la pareja — si es que la hay-. Por lo tanto, no es una decisión que la tome sólo una persona adulta, porque incluso en casos de padrastros se aúnan criterios y se actúa de manera conjunta. 
Me dan permiso los dos. Tengo que preguntarles a los dos. Le pregunto a mi madre y me dice que sí, luego le pregunto a mi padre y me dice que sí, pues ya. (Víctor, 13 años)

Históricamente, la autoridad familiar era potestad del padre, quien ejercía el poder de manera simbólica frente a las niñas y los niños, que eran cuidados por la madre. Aunque dicho estereotipo se ha matizado en la realidad, aún existen ese tipo de prácticas patriarcales en muchas familias (Brullet y Torrabadella, 2004). En nuestra investigación, encontramos un caso donde las decisiones finales sobre los permisos siguen cayendo en la figura del padre, quien es representado simbólicamente como un juez que dice la última palabra:

Decido yo mayormente digamos entre comillas, pero el que ejecuta sobre eso es el papá [...] Pero el que decide al último es el papá. Si el papá dice «no», es «no». Yo digo: «Mira hijo, parece que no». A mis hijos yo les hablo: «Si ustedes no me hacen caso, yo elevo el juicio al juez, para que les dé la sentencia» [risas], y ahí yo digo: «Él da la sentencia, ahí no me miren a mí para ningún lado, yo desaparezco». (Eugenia, 32 años)

La concesión de los permisos también está relacionada con el orden vigente, ya que tiene que ver con las libertades que son accesibles en base a la edad y el género de las personas. En el siguiente relato de una niña, vemos la interconexión de las dimensiones de género y edad cuando ella reflexiona sobre las desigualdades de los horarios de salida entre ella y su hermano:

A veces, a él le dejan salir más horas, pero me parece que es porque tiene más edad, y aunque vayamos con grupos y en el mismo grupo, me refiero al grupo de los amigos y vamos allí, a él lo dejan más tiempo que a mí [...] Él es cuatro años mayor que yo. (Julieta, 13 años)

La otra dimensión del ejercicio de la autoridad parental recae en las sanciones cuando ocurren situaciones que, desde el punto de vista adulto, requieren una corrección. Las niñas y los niños entrevistados en este estudio declaran que sus faltas son castigadas de diversas maneras, entre las que predominan las amenazas verbales, la privación de objetos electrónicos, impedirles asistir a ciertas actividades familiares o salir con las amistades. El castigo más común es privarles de lo que más les gusta.

Esta capacidad de dar permisos y de imponer castigos precisa legitimidad para desarrollarse efectivamente. Las hijas y los hijos entrevistados en este estudio y que han sido reagrupados nos comentan que ellos deben aprenden a reconocer la autoridad de sus madres y sus padres, la cual se ha vivido de una manera más simbólica que concreta durante el tiempo de vinculación transnacional, y que, además, en Perú respetaban otras «autoridades», como la de las abuelas o las tías que les cuidaban. Por su parte, los adultos también se deben re-acomodar para llegar a acuerdos familiares, donde ineludiblemente 
se hacen negociaciones. Por lo tanto, es un proceso de reacomodación recíproca por ambas partes:

Al principio como que choca un poco, haber estado siempre acostumbrados a que mayormente hablábamos por... Nuestras decisiones las tomábamos nosotros en Perú, sólo consultábamos acá. Si nos daban, bien; si no, también. Pero ahora llegamos acá y ahora tenemos que consultar. Andan detrás de nosotros y todo eso. Uno está acostumbrado a tomar decisiones solo. (Adrián, 17 años)

En el relato recién expuesto, vemos que la legitimidad de la autoridad adulta está condicionada al período de separación física y, por ello, en el momento de la reagrupación, se reconoce «un choque» generacional. Durante el tiempo que el niño estuvo en Perú a cargo de otras personas, él junto a sus hermanos pudieron experimentar cierta autonomía respecto al poder adulto («nuestras decisiones las tomábamos nosotros»), mientras que, una vez se reencuentran con su madre en Barcelona, especialmente él siente que debe volver a negociar y a legitimar la autoridad materna. El niño siente que su madre mantiene una actitud de excesivo cuidado al «andar detrás de él», lo que puede ser una forma que tiene la madre para compensar los años de separación física.

Desde el punto de vista de la distribución del trabajo doméstico, en las familias peruanas reagrupadas en Barcelona y participantes en este estudio, constatamos que se distribuye de manera medianamente participativa. No es extraño que las niñas y los niños declaren cooperar con pequeñas y medianas tareas domésticas durante el tiempo que residían en Perú, puesto que, como ya vimos, son proveedores de bienestar familiar junto con las mujeres (Gaitán, 2006b). Los relatos de nuestras entrevistas muestran que, en las familias peruanas, todos los miembros del grupo, en su medida, colaboran en las funciones hogareñas. Pero, en general, se concibe que es una responsabilidad de la madre, la cual es «ayudada" por el padre y los otros miembros del hogar:

Conforme dice uno, hay que limpiar. A mí me toca siempre limpiar mi habitación y el patio. Yo barro eso. Mi padre barre o plancha o todo, y mi madre, lo que tenga que hacer. (Juan, 14 años)

En las entrevistas, se pueden apreciar algunas diferencias de género en cuanto a la distribución doméstica. Las niñas sienten que los quehaceres asociados a la cocina siguen estando bajo su responsabilidad; mientras que, por su parte, los niños asumen que desde pequeños han cooperado en el hogar y se inclinan más por las labores de limpieza.

Sí, hay diferencia en la cocina. Mi madre siempre quiere que le ayude, pero a veces también mi hermano le ayuda. A veces yo siento que le ayudo más a mi madre en algunas cosas que sean de cocina... Todo lo que tenga que ver con cocina y mi hermano más en la limpieza. (Julieta, 13 años) 
Por otro lado, vimos algunos casos en que la familia pertenecía a la clase media en Perú y contrataba los servicios de limpieza de una mujer de clase baja. Sin embargo, una vez que el grupo familiar emigra, se pierde la posición de clase original y todos sus miembros deben colaborar con el quehacer del hogar, porque en Barcelona no se pueden pagar los servicios de limpieza.

\section{Conclusiones}

En la presente investigación, optamos por el acercamiento conceptual hacia la infancia inmigrante con actoría social, a fin de conocer la experiencia migratoria a partir de la perspectiva generacional de los sujetos que la viven. Desde el punto de vista metodológico, intentamos visibilizar la presencia infantil en los procesos migratorios utilizando entrevistas individuales que nos permitieran conocer los significados que los propios sujetos dan a los fenómenos que viven, una de las ventajas de la metodología cualitativa.

En nuestra investigación, encontramos cierta tendencia hacia el diálogo familiar respecto a las cuestiones migratorias. Aunque las niñas y los niños sienten que sí opinan en el proyecto migratorio y en la reagrupación familiar, esta participación es vista de modo ambivalente por las madres y los padres que entrevistamos. Si bien ellos identifican algunos ámbitos donde las niñas y los niños opinan, ello no significa una pauta permanente en las familias peruanas entrevistadas y reunidas en Barcelona. Por lo tanto, sería preciso que se generara un proceso inclusivo permanente en las cuestiones migratorias y familiares, lo que facilitaría reconocer a las niñas y a los niños como verdaderos sujetos sociales, y esto ayudaría a dejar de tratarles como objetos.

Respecto a las manifestaciones del poder en las relaciones generacionales, observamos que, en las familias peruanas entrevistadas, la autoridad adulta necesariamente se modifica una vez que sus integrantes se reagrupan en Barcelona. Específicamente, los permisos y las sanciones infantiles continúan siendo una cuestión decidida por las personas adultas del grupo familiar, y las sanciones terminan siendo casi siempre "quitarles lo que más les gusta» a las niñas y a los niños. Por último, respecto a las relaciones de género y a la distribución del trabajo del hogar, en nuestras entrevistas observamos que la labor doméstica sigue siendo vista como una responsabilidad eminentemente de las mujeres y las niñas, aunque los niños y los adolescentes también señalan que cooperan en diversas tareas caseras.

Sin duda, es un gran desafío para las familias peruanas entrevistadas en este estudio y que han vivido procesos de reagrupación familiar en Barcelona, no sólo reconstruir los vínculos filiales que se han visto indudablemente afectados por la migración, sino también hacerlo de una manera que facilite un proceso inclusivo de participación infantil.

El presente trabajo constituye un primer acercamiento hacia la realidad de los hogares peruanos que participan en procesos migratorios de reagrupación familiar en Barcelona, por lo tanto, los datos y la información presentados aquí 
tienen un alcance exploratorio. En la tesis doctoral, es indispensable continuar profundizando en los ámbitos referidos a la participación infantil en dichos procesos y en las desigualdades de género que se dan en los mismos, también es necesario incorporar elementos de la posición de clase social de las familias, lo que nos ayuda a contextualizar los proyectos migratorios de las familias peruanas y la forma en que estas cuestiones inciden específicamente en las niñas y los niños migrantes en Barcelona.

\section{Bibliografía}

ALTAMirano, Teófilo (2000). Liderazgo y organizaciones de peruanos en el exterior: culturas transnacionales e imaginarios sobre el desarrollo. Vol. 1. Lima: Pontificia Universidad Católica del Perú.

APARICIO, Rosa y TORNOS, Andrés (2006). Hijos de inmigrantes que se hacen adultos: marroquies, dominicanos y peruanos. Madrid: Ministerio de Trabajo y Asuntos Sociales.

ARIÉS, Philippe (1987). El niño y la vida familiar en el antiguo régimen. Madrid: Taurus.

BRULLET, Cristina y TORRABADELla, Laura (2004). «La infancia en las dinámicas de transformación familiar». En: GÓMEZ-GRANELL, Carme et al. (coords.). Infancia y familias: realidades y tendencias. Barcelona: Ariel-CIIMU, 37-61.

BRYCESON, Deborah y VuORELA, Ulla (eds.) (2002). The transnational family: New European frontiers and global networks. Oxford: Berg.

CARRASCO, Silvia (2004a). «Infancia e inmigración: proyectos y realidades». En: GOMEZGRANELL, Carme et al. (coords.). Infancia y familias: realidades y tendencias. Barcelona: Ariel-CIIMU, 205-231.

- (2004b). Inmigración, contexto familiar y educación: Procesos y experiencias de la población marroquí, ecuatoriana, china y senegambiana. Bellaterra (Barcelona): Universitat Autònoma de Barcelona. Institut de Ciències de l'Educació.

ECKSTEIN, Susan (2002). "On deconstructing and reconstructing the meaning of immigrant generations». En: LeVITT, Peggy y WATERS, Mary. (eds.) (2002). The changing face of home: The transnational lives of the second generation. Nueva York: Russell Sage Foundation, 211-215.

ESCRIVÁ, María Àngels (2000). «:Empleadas de por vida? Peruanas en el servicio doméstico de Barcelona». Papers, 60, 328-342.

GAITÁN, Lourdes (2006a). «La nueva sociología de la infancia: Aportaciones de una mirada distinta». Política y Sociedad, 43 (1), 9-26.

- (2006b). Sociología de la infancia. Madrid: Síntesis.

- (dir.) (2008). Los niños como actores en los procesos migratorios: Implicaciones para los proyectos de cooperación. Madrid: Universidad Complutense de Madrid.

GARCíA BORREGO, Iñaki (2008). Herederos de la condición inmigrante: adolescentes y jóvenes en familias madrileñas de origen extranjero. Tesis doctoral. Madrid: Departamento de Sociología I. UNED.

GÓMEZ-Granell, Carme et al. (2004). «Introducción». En: GÓMEZ-Granell, Carme et al. (coords.). Infancia y familias: realidades y tendencias. Barcelona: Ariel-CIIMU, $15-32$.

LABRADOR FERNÁNDEZ, Jesús (2001). Identidad e inmigración: Un estudio cualitativo con inmigrantes peruanos en Madrid. Madrid: Universidad Pontificia de Comillas. 
LaWs, Sophie y ManN, Gillian (2004). ¿Asi que quiere hacer participar a los niños y niñas en la investigación?: Paquete de herramientas para apoyar la participación significativa y ética de los niños y niñas en la investigación relativa a la violencia contra los niños y niñas. Estocolmo: Save the Children-Suecia.

LeVITT, Peggy y Waters, Mary (eds.) (2002). The changing face of home: The transnational lives of the second generation. Nueva York: Russell Sage Foundation.

LiEBEL, Manfred (2007). Entre protección y emancipación: Derechos de la infancia y politicas sociales. Madrid: Experto en Políticas Sociales de Infancia-UCM.

Mannheim, Karl (1928). «Das Problem der Generationen». Kölner Vierteljahreshefte for Soziologie, VII, 2. Versión utilizada: «El problema de las generaciones». Revista Española de Investigaciones Sociológicas, 62, 1993, 193-200.

PARELla, Sònia (2007). "Los vínculos afectivos y de cuidado en las familias transnacionales: Migrantes ecuatorianos y peruanos en España». Migraciones Internacionales, 4 (2), 151-188.

Pedone, Claudia (2003). "Tú siempre jalas a los tuyos»: Cadenas y redes migratorias de familias ecuatorianas hacia España. Tesis doctoral. Barcelona: Departamento de Geografía. Universitat Autònoma de Barcelona.

- (2007). "Lo de migrar me lo tomaría con calma»: Representaciones sociales de jóvenes en torno al proyecto migratorio familiar. Ponencia presentada en el Seminario Internacional de Investigación sobre "Migraciones, Familias y Transnacionalidad", Universidad de Murcia (España), FLACSO-Ecuador, Universidad de Cuenca (Ecuador) y Universidad de Buenos Aires (Argentina), Murcia, octubre de 2007.

PORTES, Alejandro (ed.) (1996). The new second generation. Nueva York: Russell Sage Foundation.

PORTES, Alejandro y Rumbaut, Rubén (2001). Legacies: The story of the immigrant second generation. Berkeley: University of California Press.

Portes, Alejandro; FernándeZ-Kelly, Patricia y Halles, William (2006). "La asimilación segmentada sobre el terreno: la nueva segunda generación al inicio de la vida adulta». Migraciones, 19, 7-58.

RodríGueZ, Iván (2007). Para una sociología de la infancia: aspectos teóricos y metodológicos. Madrid: Centro de Investigaciones Sociológicas.

Solé, Carlota; PARella, Sònia y CalvanCANTI, Leonardo (2007). Los vínculos económicos y familiares transnacionales: Los inmigrantes ecuatorianos y peruanos en España. Madrid: BBVA.

SuÁrez-Orozco, Carola y SuÁrez-Orozco, Marcelo (2003). La infancia de la inmigración. Madrid: Morata.

TERren, Eduardo y CARrasco, Cristina (2007). «Familia, escuela e inmigración». Migraciones, 22, 9-46.

Torrabadella, Laura y Tejero, Esther (2005). Pioneres i pioners: Trajectòries biogràfiques de filles y fills de famílies immigrades a Catalunya. Barcelona: Fundació Jaume Bofill. Fundació Caixa de Sabadell. 